\title{
Bayesian conditional autoregressive models to assess spatial patterns of diarrhoea risk among children under the age of 5 years in Mbour, Senegal
}

\author{
Sokhna Thiam, ${ }^{1-3}$ Guéladio Cissé, ${ }^{1,2}$ Anna-Sofie Stensgaard, ${ }^{4}$ Aminata Niang-Diène, ${ }^{5}$ \\ Jürg Utzinger, ${ }^{1,2}$ Penelope Vounatsou ${ }^{1,2}$ \\ ${ }^{1}$ Swiss Tropical and Public Health Institute, Basel, Switzerland; ${ }^{2}$ University of Basel, Basel, Switzerland; \\ ${ }^{3}$ Health Research, Epidemiological Surveillance and Training Institute, Dakar, Senegal; ${ }^{4}$ Center for \\ Macroecology, Evolution and Climate, GLOBE Institute, University of Copenhagen, Copenhagen, \\ Denmark; ${ }^{5}$ Department of Geography, University Cheikh Anta Diop of Dakar, Dakar, Senegal
}

\begin{abstract}
Diarrhoeal diseases remain a major public health problem, causing more than half a million child deaths every year, particularly in low- and middle-income countries (LMICs). Despite exist-
\end{abstract}

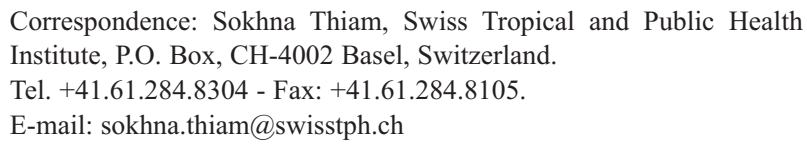

Key words: Bayesian conditional autoregressive models; Diarrhoea; Risk profiling; Senegal; Spatial pattern.

See online Appendix for additional tables.

Acknowledgements: the authors are grateful to all the study participants for their kind cooperation and participation in the survey. We also thank all the field enumerators for their support during data collection.

Contributions: ST, GC, ASS, AND, JU and PV designed the study. ST, GC and AND coordinated the field data collection. ST and PV did the statistical analysis. ST wrote the first draft of the manuscript. GC, ASS, AND, JU and PV reviewed the manuscript. All authors read and approved the final version of the manuscript prior to submission.

Conflict of interest: the authors declare no potential conflict of interest.

Funding: this study was supported by funding from the Swiss Government, through the Swiss Government Excellence Scholarships for Foreign Scholars (ESKAS scholarship) and the Swiss AfricanResearch Cooperation (SARECO) programme. ASS is grateful to Knud Højgaards Fond for supporting the Research Platform for Disease Ecology, Climate and Health and thank the Danish National Research Foundation for its support of the Center for Macroecology, Evolution and Climate (grant no. DNRF96).

Received for publication: 12 October 2019.

Revision received: 21 October 2019.

Accepted for publication: 21 October 2019.

${ }^{\circ}$ Copyright: the Author(s), 2019

Licensee PAGEPress, Italy

Geospatial Health 2019; 14:823

doi:10.4081/gh.2019.823

This article is distributed under the terms of the Creative Commons Attribution Noncommercial License (CC BY-NC 4.0) which permits any noncommercial use, distribution, and reproduction in any medium, provided the original author(s) and source are credited. ing knowledge on the aetiologies and causes of diarrhoeal diseases, relatively little is known about its spatial patterns in LMICs, including Senegal. In the present study, data from a cross-sectional survey carried out in 2016 were analysed to describe the spatial pattern of diarrhoeal prevalence in children under the age of 5 years in the secondary city of Mbour in the south-western part of Senegal. Bayesian conditional autoregressive (CAR) models with spatially varying coefficients were employed to determine the effect of sociodemographic, economic and climate parameters on diarrhoeal prevalence. We observed substantial spatial heterogeneities in diarrhoea prevalence. Risk maps, stratified by age group, showed that diarrhoeal prevalence was higher in children aged 25-59 months compared to their younger counterparts with the highest risk observed in the north and south peripheral neighbourhoods, especially in Grand Mbour, Médine, Liberté and Zone Sonatel. The posterior relative risk estimate obtained from the Bayesian CAR model indicated that a unit increase in the proportion of people with untreated stored drinking water was associated with a $29 \%$ higher risk of diarrhoea. A unit increase in rainfall was also associated with an increase in diarrhoea risk. Our findings suggest that public health officials should integrate disease mapping and cluster analyses and consider the varying effects of sociodemographic factors in developing and implementing areaspecific interventions for reducing diarrhoea.

\section{Introduction}

Diarrhoea remains an important public health problem that is associated with high childhood mortality and morbidity, particularly in low- and middle-income countries (LMICs). In 2016, diarrhoea was the eighth leading cause of death among all ages and the fifth leading cause of death among children under the age of 5 years (GBD 2016 Diarrhoeal Disease Collaborators, 2018). Although the number of deaths due to diarrhoea among children under the age of 5 years decreased by an estimated $40.6 \%$ between 2007 and 2017, it is still estimated that, in 2017, more than half a million children of this age group died due to diarrhoea (GBD 2017 Causes of Death Collaborators, 2018). Diarrhoea-related morbidity has also declined over the past several years (Fischer Walker et al., 2012; GBD Diarrhoeal Diseases Collaborators, 2017). There are nearly 1.7 billion episodes of diarrhoea globally every year (Walker et al., 2013; WHO, 2017). Most of diarrhoearelated deaths are attributable to unsafe water, inadequate sanitation and lack of personal hygiene (Black et al., 2003; GBD Diarrhoeal Diseases Collaborators, 2017). Indeed, in a comprehensive review of the literature, the World Health Organization 
(WHO) estimated that $58 \%$ of all cases of diarrhoea in LMICs could be attributed to inadequate drinking water (34\%), sanitation (19\%) and hygiene (20\%) (Prüss-Üstun et al., 2014).

In Senegal, diarrhoeal diseases continue to cause considerable mortality and morbidity. The highest burden in terms of disabilityadjusted life years (DALYs) among children under the age of 5 years is still caused by diarrhoeal diseases. Even though the burden of diarrhoeal diseases decreased by 6\% between 1990 and 2016 across Senegal, it is still responsible for $7 \%$ of all child deaths and an estimated 325,335 DALYs $(13,776$ DALYs per 100,000 children under the age of 5 years) (GBD 2015 DALYs and HALE Collaborators, 2016; Institute for Health Metrics and Evalutation, 2016). In a recent study conducted in the secondary city of Mbour, the reported diarrhoeal prevalence at household level among children under 5 years of age was $26.0 \%$ (Thiam et al., 2017a). This high burden of diarrhoea is reported despite improvement in access to drinking water and sanitation.

Previous studies in Senegal have mainly focused on clinical features of diarrhoea and looked at single geographic units (Sambe-Ba et al., 2013; Sire et al., 2013). Hence, there is an important knowledge gap with respect to the spatial patterns of diarrhoea, because prior studies did not identify existing areas of priority for intervention. It is important to note that diarrhoea morbidity varies across geographical areas; some areas are likely to sustain high morbidity over time due to unplanned rapid urbanisation without improvement on essential amenities (e.g. access to clean water and improved sanitation), which do not meet the demands of the rising population (Osei and Stein, 2017a). Hence, it is crucial to identify areas with high risk of diarrhoea, in order to assist decision makers in the spatial targeting of interventions and monitor progress over time. Since children are the most vulnerable group to suffer from diarrhoea, information on age-specific patterns are important to meet the Sustainable Development Goal 3 (SDG 3) that is to ensure healthy lives and promote wellbeing for all at all ages.

This paper aims to map the spatial pattern of diarrhoeal prevalence among children under the age of 5 years in the secondary city of Mbour, located in the south-western part of Senegal. Our paper complements prior research pertaining to knowledge, attitudes and practices of diarrhoea in this urban setting of Senegal (Thiam et al., 2019). By using a Bayesian conditional autoregressive (CAR) modelling approach, we specifically assess the effect of sociodemographic, economic and climatic factors that might govern diarrhoea morbidity.

\section{Materials and Methods}

\section{Ethical approval}

This study received ethical approval from the national research ethics committee (Comité National d'Ethique de la RechercheCER) of Senegal (reference no.: 0106/2015/CER/UCAD). An interview was conducted only if the respondent provided his or her informed consent. Given the high illiteracy rate of mothers and caregivers of under 5-year-old children in Mbour, we aimed for verbal rather than written informed consent. The interviewer signed his or her name attesting to the fact that he/she read the consent statement to the respondent

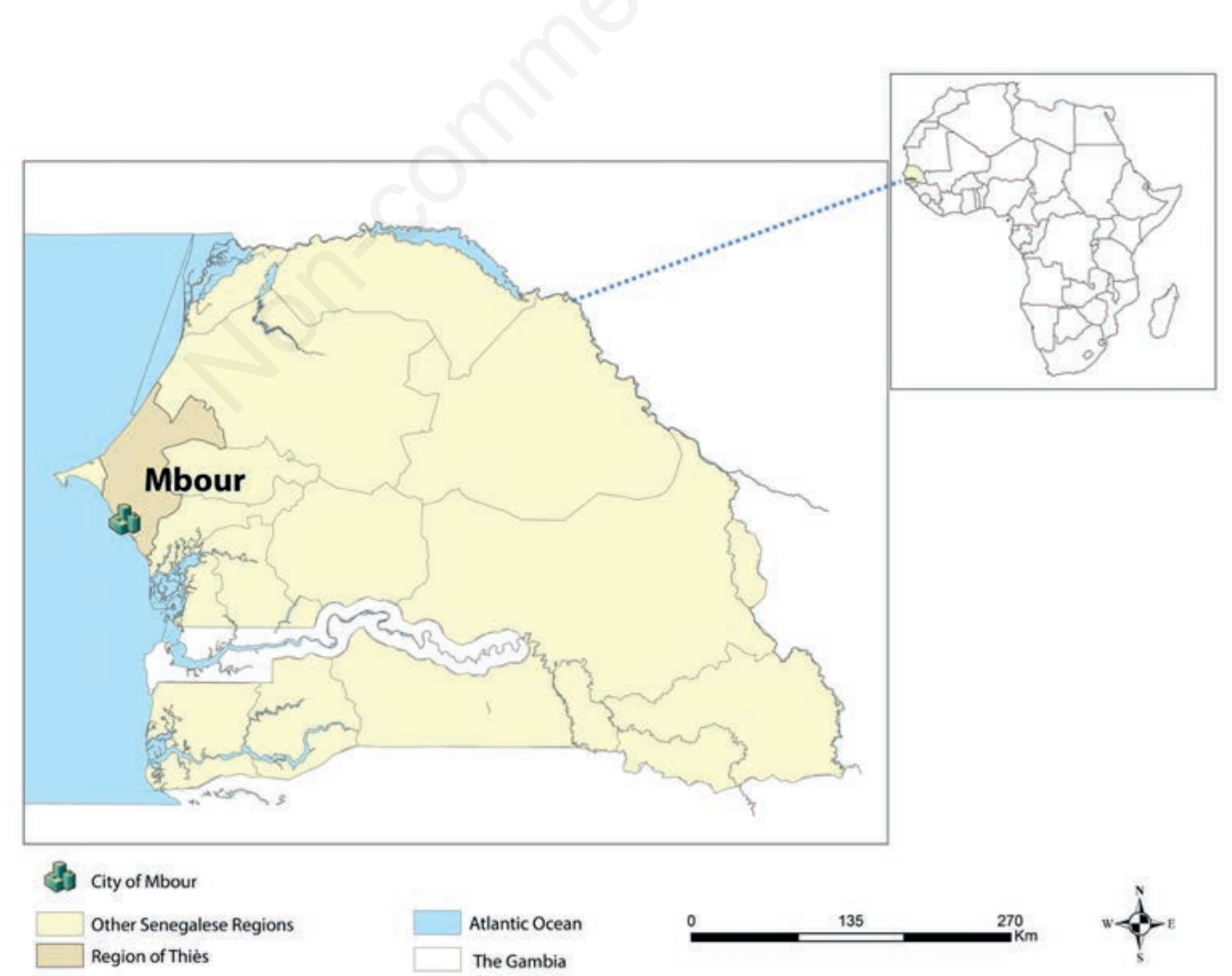

Figure 1. Map showing the administrative region and the location of Mbour in Senegal. 


\section{Study area and design}

The study was conducted in the secondary city of Mbour, which is located in the south-western part of Senegal in the Region of Thiès (Figure 1). Mbour consists of 25 neighbourhoods. A crosssectional survey was carried out in September and October 2016, covering all 25 neighbourhoods. The outcome of interest was diarrhoeal disease case report among under 5-year-old children in the sampled household. A detailed description of the activities performed during the cross-sectional survey has been published elsewhere (Thiam et al., 2017b).

\section{Sociodemographic, economic and climate data}

As diarrhoea transmission is known to be influenced by several factors, including water, sanitation and hygiene (WASH), climatic, sociodemographic and economic factors, we obtained sociodemographic and economic covariates from a survey questionnaire. We constructed the following sociodemographic variables as risk factors of diarrhoea: unsafe drinking water source $(u d w s)$, untreated stored drinking water $(u s d w)$, unsafe liquid waste disposal ( $u l w d)$ and unsafe solid waste disposal ( $u s w d$ ). We defined the socio-environmental variables as follows: i) $u d w s$ as the proportion of the neighbourhood's surveyed households that lack access to pipeborne water (either in dwellings, outside dwellings or public standpipes); ii) $u s d w$ as the proportion of the neighbourhood's surveyed households that do not treat their stored drinking water; iii) $u l w d$ as the proportion of the neighbourhood's surveyed households that dispose liquid waste in the street; and iv) uswd as the proportion of the neighbourhood's surveyed households that do not have a waste bin. An economic status variable was derived from principal component analysis (PCA) as a weighted sum of household assets that was included in the analysis as a continuous covariate. A detailed description of the household assets used to run the PCA has been described in a previous paper (Thiam et al., 2017a).

Climatic covariates were obtained from readily available remote sensing sources (Table 1). The climatic factors used in our analysis are land surface temperature (LST) at day and night, altitude and rainfall covering the survey period (i.e. September and October 2016). LST and altitude data were extracted at the unit of the neighbourhood from the Moderate Resolution Imaging Spectroradiometer (MODIS) database. Rainfall estimates data were downloaded from the Africa Data Dissemination Service database. Climatic covariates were included in the analysis as continuous covariates. The data were aggregated by neighbourhood units and the geographical scale of the analyses considered the entire 25 neighbourhoods of Mbour.

\section{Bayesian conditional autoregressive modelling approach}

First, a preliminary binomial logistic regression of diarrhoea cases among children under the age of 5 years was performed to select covariates. Four climatic variables (i.e. $\mathrm{LST}_{\text {Day }}, \mathrm{LST}_{\mathrm{Night}}$, rainfall and altitude) and four sociodemographic and economic variables (i.e. $u d w s, u s d w$, ulwd and uswd) were included as covariates. Second, we fitted three separate logistic regression models to estimate the effect of sociodemographic, economic and climatic parameters on diarrhoea morbidity in our target group of children using a Bayesian framework. The first model included only climatic covariates; the second model included sociodemographic and economic covariates, whereas the third model contained all of the components of models 1 and 2, as well as a spatially structured random effect. The spatially structured random effect was formulated assuming a CAR model prior distribution (Cressie, 2015; Ssempiira et al., 2017), which introduces a neighbours-based spatial structure random effect for the regression coefficients (Bivand et al., 2013). To adjust for spatial correlation present in diarrhoea data due to similar exposure effect in neighbouring areas, cluster-specific random effects were added to each model to account for unknown or unmeasured risk factors by introducing an extra source of variability into the model. This approach was employed to generate spatial weights matrix, which assigned value 1 to areas that share borders and 0 otherwise. More specifically, for the third model, it was assumed that $Y_{i j}$ is a binary outcome taking value 1 or 0 if a child $i$ at neighbourhood $s_{j}$ had diarrhoea in the 2 weeks prior to the survey. $Y_{i j}$ is assumed to follow a Bernoulli distribution $Y_{i \mathrm{j}} \sim B n\left(\mathrm{~N}_{\mathrm{ij}}, P_{\mathrm{ij}}\right)$ and is related to its predictors using a logistic regression model, as follows (Eq. 1):

$\operatorname{logit}\left(p_{i j}\right)=\beta_{0}+\sum_{k=1}^{k} \beta_{k} X_{i j}^{k}+\phi_{j}+\omega_{i}$,

Eq. 1

where $p_{i j}$ is the diarrhoea case at neighbourhood $i, s_{j}$ of having diarrhoea (i.e. an offset to control for population size), and $\beta=\left(\beta_{0}\right.$, $\left.\beta_{l}, \ldots, \beta_{k}\right)$ is the vector of $k$ regression coefficients. Spatial dependence is introduced by adding location-specific random effects $\phi_{j}$ at every surveyed location $\left(s_{j}\right)$. Non-spatial variation is estimated by the random effects $\omega_{i} \sim N\left(0, \sigma_{w}^{2}\right)$ for the unstructured spatial effect assumed independent and normally distributed with mean 0 and variance $\sigma_{w}^{2}$ for the spatially structured random effect. A CAR prior distribution was employed to model the spatially structured random effect. A flat prior distribution was specified for the intercept, while a non-informative normal prior distribution was used for the coefficients. The prior for the precision of the spatially structured random effects was specified using non-informative gamma distribution with shape and scale parameters equal to 0.5 . An initial burn-in of 1,000 iterations was run, and these iterations were discarded. Subsequent blocks of 250,000 iterations were run and checked for convergence. Convergence was assessed by visual inspection of posterior density and history plots. All model parameters were stored and summarised for the analysis. In all analyses, a level of 0.05 was adopted to indicate statistical significance as indicated by a $95 \%$ Bayesian credible interval (BCI) for the relative risk excluding 1 . Continuous covariates were standardised for

Table 1. Remote sensing data sources, reporting period and spatial resolution.

\begin{tabular}{lccc} 
Remote sensing data source & Type & Period & Spatial resolution \\
MODIS & LST $_{\text {Day }}$ & September/October 2016 & $1 \times 1 \mathrm{~km}$ \\
& $\mathrm{LST}_{\text {Night }}$ & September/October 2016 & $1 \times 1 \mathrm{~km}$ \\
USGS/decadal RFE & RFE & September/October 2016 & $80 \times 80 \mathrm{~km}$ \\
\hline SRTM-Altitude & Altitude & September/October 2016 & $90 \times 90 \mathrm{~km}$ \\
\hline
\end{tabular}

MODIS, Moderate Resolution Imaging Spectroradiometer (http://modis.gsfc.nasa.gov); USGS/decadal RFE, United States Geographical Survey/decadal rainfall estimates (http://earlywarning.usgs.gov); SRTM-Altitude, Shuttle Radar Topography Mission (http:/www.cgiar-csi.org/data/srtm-90m-digital-elevation-database-v41); LST, land surface temperature. 
addressing correlation between covariates. Parameter estimates were summarised using posterior medians and the corresponding $95 \%$ BCI. For epidemiological interpretation, model estimates were exponentiated to produce odds ratios (ORs).

Model fits and parameter estimation were performed using a Bayesian framework and Markov chain Monte Carlo (MCMC) estimation. Model specification was completed by assigning gamma prior distributions to model parameters. Data analysis was carried out in STATA version 14 (StataCorp; College Station, TX, USA). OpenBUGS version 3.2.3 (Imperial College and Medical Research Council; London, UK) was used to perform model fit. The maps were produced using ArcGIS software version 10.5 (ArcMap, ESRI; Redlands, CA, USA).

\section{Results}

\section{Summary of sociodemographic and WASH conditions of the surveyed households}

A total of 1,083 children under the age of 5 years from 761 households participated in the cross-sectional survey conducted in Mbour, between September and October 2016. Sociodemographic and economic characteristics of the surveyed households and WASH conditions in the four stratified zones of Mbour are provided as supplementary files (Appendix Tables A1 and A2).

\section{Distribution of the observed diarrhoeal prevalence and the sociodemographic covariates}

The observed diarrhoea prevalence in children under the age of 5 years, stratified by age group, zone and neighbourhoods, are summarised in Table 2. The number of children among those surveyed who reportedly had diarrhoea during the 2 weeks preceding the survey was mapped by neighbourhood areas to illustrate the distribution patterns of the observed prevalence (Figure 2).

Table 2 shows that the overall observed diarrhoea prevalence of $33.9 \%$ varied between $20.0 \%$ (neighbourhood of Grand Mbour) and $57.9 \%$ (neighbourhood of Baye Deuk). Among the youngest age group (children aged below 12 months), the observed diarrhoeal prevalence was $31.6 \%$, varying between $8.6 \%$ (neighbourhood of Zone Résidentielle) and 42.9\% (neighbourhood of Mbour Sérère Souf). For children aged 12-24 months, the overall prevalence of diarrhoea was $40.0 \%$, varying between $14.3 \%$ (neighbourhood of Mbour Sérère Souf) and 57.9\% (neighbourhood of Baye Deuk). For children aged 36-59 months, the observed prevalence was $29.3 \%$, varying between $21.0 \%$ (neighbourhood of Baye Deuk) and 53.1\% (neighbourhood of Medine). The geographical distribution of the observed prevalence, stratified by age group, is illustrated by the maps displayed in Figure 2.

The spatial distribution of the model-based estimation of diarrhoea risk from the posterior estimates, adjusted for spatially random effect, spatial correlation and varying coefficient effects, are shown in Figure 3. Most of the neighbourhoods in Mbour exhibit

Table 2. Summary of the observed diarrhoeal prevalence in children under the age of 5 years, stratified by age group, zone and neighbourhood in Mbour in late 2016.

\begin{tabular}{|c|c|c|c|c|c|}
\hline Zone & Neighbourhood & $<5$ years $(\%)$ & $\begin{array}{l}\text { rved diarrhoeal pr } \\
<12 \text { months }(\%)\end{array}$ & $\begin{array}{l}\text { in children aged } \\
12-24 \text { months }(\%)\end{array}$ & 36-59 months (\%) \\
\hline UCA & $\begin{array}{l}\text { Château d'eau Nord } \\
\text { Château d'eau Sud } \\
\text { Onze Novembre } \\
\text { Tefess } \\
\text { Golf } \\
\text { Zone Residentielle } \\
\text { Mbour Serère Souf }\end{array}$ & $\begin{array}{l}38.3 \\
30.6 \\
27.6 \\
33.3 \\
57.1 \\
38.5 \\
54.3 \\
28.6\end{array}$ & $\begin{array}{c}21.1 \\
19.4 \\
31.0 \\
20.5 \\
32.1 \\
15.4 \\
8.6 \\
42.9\end{array}$ & $\begin{array}{l}38.3 \\
38.7 \\
27.6 \\
43.6 \\
39.3 \\
38.5 \\
42.9 \\
14.3\end{array}$ & $\begin{array}{l}40.5 \\
41.9 \\
41.4 \\
35.9 \\
28.6 \\
46.2 \\
48.6 \\
42.9\end{array}$ \\
\hline PCA & $\begin{array}{l}\text { Darou Salam } \\
\text { Diamaguene } 1 \\
\text { Diamaguene } 2 \\
\text { Baye Deuk } \\
\text { Santessou } \\
\text { Thiocé Est } \\
\text { Thiocé Ouest } \\
\text { Mbour Toucouleur } \\
\text { Mbour Serère Kaw }\end{array}$ & $\begin{array}{l}34.8 \\
30.6 \\
35.6 \\
34.7 \\
57.9 \\
31.8 \\
32.4 \\
36.4 \\
32.3 \\
36.4\end{array}$ & $\begin{array}{l}22.5 \\
16.1 \\
19.2 \\
27.7 \\
21.1 \\
18.2 \\
18.9 \\
33.3 \\
22.6 \\
27.3\end{array}$ & $\begin{array}{l}39.5 \\
40.3 \\
47.9 \\
35.6 \\
57.9 \\
40.9 \\
36.5 \\
33.3 \\
41.9 \\
30.3 \\
\end{array}$ & $\begin{array}{l}37.9 \\
43.5 \\
32.9 \\
36.6 \\
21.1 \\
40.9 \\
44.6 \\
33.3 \\
35.5 \\
42.4 \\
\end{array}$ \\
\hline NPA & $\begin{array}{l}\text { Grand Mbour } \\
\text { Liberté } \\
\text { Médine } \\
\text { Santhie }\end{array}$ & $\begin{array}{l}26.9 \\
20.0 \\
31.6 \\
26.6 \\
31.4\end{array}$ & $\begin{array}{l}15.5 \\
12.5 \\
10.5 \\
15.6 \\
27.5\end{array}$ & $\begin{array}{l}36.2 \\
38.8 \\
36.8 \\
31.3 \\
37.3\end{array}$ & $\begin{array}{l}48.3 \\
48.8 \\
52.6 \\
53.1 \\
35.3\end{array}$ \\
\hline SPA & $\begin{array}{l}\text { Gouye Mouride } \\
\text { Oncad } \\
\text { Mbour Maure } \\
\text { Zone Sonatel }\end{array}$ & $\begin{array}{l}37.2 \\
32.4 \\
34.6 \\
33.3 \\
46.2\end{array}$ & $\begin{array}{l}13.1 \\
10.8 \\
15.4 \\
22.2 \\
10.3\end{array}$ & $\begin{array}{l}42.3 \\
37.8 \\
46.2 \\
44.4 \\
41.0\end{array}$ & $\begin{array}{l}44.5 \\
51.4 \\
38.5 \\
33.3 \\
48.7\end{array}$ \\
\hline Overall & & 33.9 & 31.6 & 40.0 & 29.3 \\
\hline
\end{tabular}

UCA, Urban Central Areas; PCA, Peri-Central Areas; NPA, Northern Peripheral Areas; SPA, Southern Peripheral Areas. 
similar patterns, except four neighbourhoods with diarrhoea risk above $40 \%$.

A summary of the sociodemographic covariates can be seen in Table 3. Briefly, the proportion of the surveyed household without safe drinking water was $41.7 \%$, varying from $0 \%$ in Mbour Sérère Souf to $100 \%$ in Baye Deuk and Gouye Mouride. About $69 \%$ of the surveyed households do not treat their stored drinking water; this proportion varied from $28.9 \%$ in Médine to $85.0 \%$ in Tefess. The proportion of the surveyed household without access to safe liquid waste disposal ranged from $4.2 \%$ in Thiocé Ouest to $91.7 \%$ in Zone Residentielle and Mbour Sérère Kaw. The proportion of the surveyed household without access to proper solid waste disposal ranged from $0 \%$ to $100 \%$.

\section{Posterior estimates of the effect of sociodemographic, economic and climatic factors}

The results from the Bayesian CAR models are presented in Table 4. To facilitate interpretation of the effect of sociodemographic and climatic factors on diarrhoea risk, all parameter estimates were exponentiated. In model 1 , association of diarrhoea prevalence with altitude, rainfall and $\mathrm{LST}_{\text {day }}$ were positive; however, none of these associations were of statistical importance. In model 2 , untreated stored drinking water was significantly associated with diarrhoea prevalence in children. A unit increase in the proportion of people who do not treat their stored drinking water increases diarrhoea risk by $22 \%$, suggesting that diarrhoea risk for people using untreated stored drinking water is $22 \%$ higher than those who use treated stored drinking water. The results from model 2 also indicate a significant association between diarrhoea risk and economic status. No multiplicative effects were observed for $u d w s$ and $u s w d$, meaning that differences in sources of drinking water and wastewater disposal could not account for the observed variation in diarrhoea risk across neighbourhoods. In model 3, rainfall, $\mathrm{LST}_{\text {day }}$, untreated stored drinking water, unsafe wastewater disposal and economic status were positively associated with diarrhoea prevalence in children in Mbour.

\section{Discussion}

This study aimed to explore and map the spatial pattern of diarrhoeal prevalence among children under the age of 5 years in the secondary city of Mbour, Senegal. Risk profiling was done at the unit of the neighbourhood $(n=25)$ and we specifically determined the effect of sociodemographic, economic and climatic factors. We employed a Bayesian framework with CAR models. The study is the first effort to explore the spatial patterns of diarrhoea in an urban setting of Senegal. The assumptions in the modelling approach are that the effect of sociodemographic, economic and climatic factors in neighbouring areas should be similar; whereas the effect should be increasingly dissimilar the further away areas are located from each other.
A

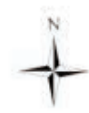

Observed prevalence (\%)

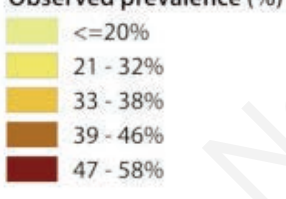

C

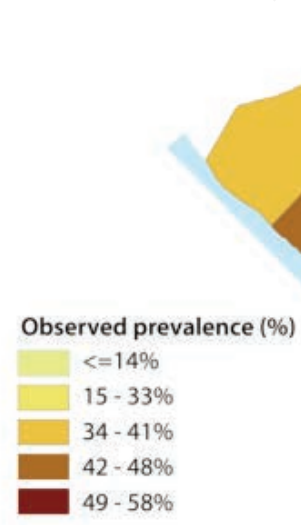

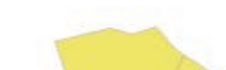

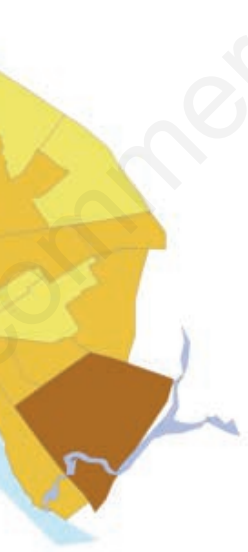

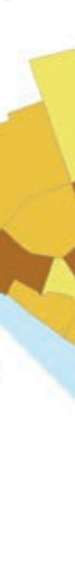
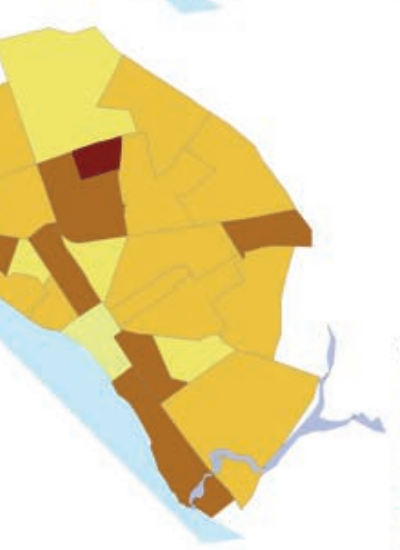

B
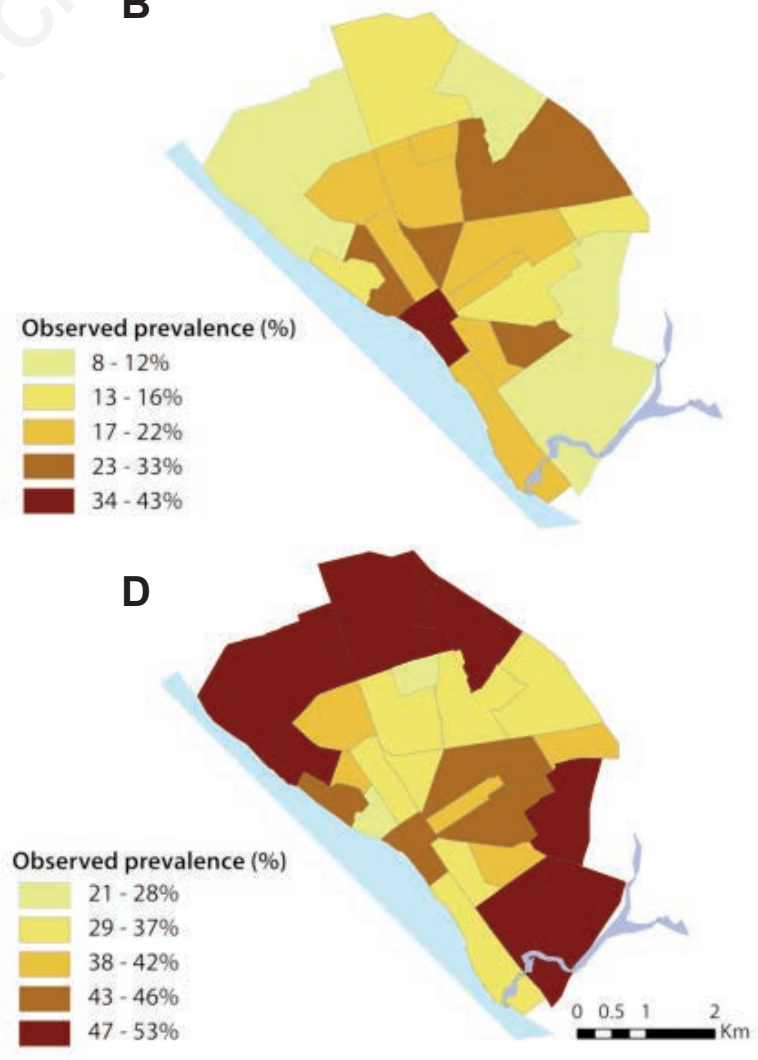

Figure 2. Spatial distribution of the observed diarrhoea prevalence in children under the age of 5 years in Mbour in 2016. Overall observed prevalence (A); observed diarrhoea prevalence among children aged 0-11 months (B), observed prevalence among children aged 12-24 months $(C)$ and observed prevalence among children aged 25-59 months (D). 
Table 3. Summary of the sociodemographic covariates, stratified by zone and neighbourhood in Mbour in late 2016.

\begin{tabular}{|c|c|c|c|c|c|}
\hline Zone & Neighbourhood & Proportion udws* (\%) & Proportion usdw ${ }^{\circ}(\%)$ & Proportion ulud ${ }^{\#}(\%)$ & Proportion uswd ( $\left.^{\S} \%\right)$ \\
\hline UCA & $\begin{array}{l}\text { Château d'eau Nord } \\
\text { Château d'eau Sud } \\
\text { Onze Novembre } \\
\text { Tefess } \\
\text { Golf } \\
\text { Zone Residentielle } \\
\text { Mbour Serère Souf }\end{array}$ & $\begin{array}{c}16.3 \\
4.8 \\
26.3 \\
7.1 \\
20.0 \\
57.1 \\
16.7 \\
0.0\end{array}$ & $\begin{array}{l}77.1 \\
76.2 \\
79.0 \\
78.6 \\
85.0 \\
78.6 \\
70.8 \\
60.0\end{array}$ & $\begin{array}{l}60.1 \\
81.0 \\
63.2 \\
39.3 \\
10.0 \\
57.1 \\
91.7 \\
60.0\end{array}$ & $\begin{array}{c}85.6 \\
95.2 \\
100.0 \\
78.6 \\
80.0 \\
100.0 \\
83.3 \\
0.0\end{array}$ \\
\hline PCA & $\begin{array}{l}\text { Darou Salam } \\
\text { Diamaguene } 1 \\
\text { Diamaguene } 2 \\
\text { Baye Deuk } \\
\text { Santessou } \\
\text { Thiocé Est } \\
\text { Thiocé Ouest } \\
\text { Mbour Toucouleur } \\
\text { Mbour Serère Kaw }\end{array}$ & $\begin{array}{c}28.9 \\
41.9 \\
22.2 \\
27.9 \\
100.0 \\
5.6 \\
17.9 \\
8.3 \\
20.0 \\
50.0\end{array}$ & $\begin{array}{l}75.8 \\
81.4 \\
83.3 \\
76.5 \\
60.0 \\
66.7 \\
71.4 \\
79.2 \\
65.0 \\
79.2\end{array}$ & $\begin{array}{c}76.7 \\
88.4 \\
72.2 \\
88.2 \\
86.7 \\
83.3 \\
76.8 \\
4.2 \\
80.0 \\
91.7\end{array}$ & $\begin{array}{c}93.2 \\
90.7 \\
90.7 \\
97.1 \\
93.3 \\
100.0 \\
94.6 \\
91.7 \\
90.0 \\
87.5\end{array}$ \\
\hline NPA & $\begin{array}{l}\text { Grand Mbour } \\
\text { Liberté } \\
\text { Médine } \\
\text { Santhie }\end{array}$ & $\begin{array}{l}59.5 \\
40.7 \\
78.3 \\
82.2 \\
37.1\end{array}$ & $\begin{array}{l}56.2 \\
66.1 \\
56.5 \\
28.9 \\
74.3\end{array}$ & $\begin{array}{l}71.9 \\
72.9 \\
76.1 \\
53.3 \\
88.6\end{array}$ & $\begin{array}{l}88.1 \\
76.3 \\
95.7 \\
93.3 \\
91.4\end{array}$ \\
\hline SPA & $\begin{array}{l}\text { Gouye Mouride } \\
\text { Oncad } \\
\text { Mbour Maure } \\
\text { Zone Sonatel }\end{array}$ & $\begin{array}{c}88.1 \\
100.0 \\
92.5 \\
37.5 \\
85.7\end{array}$ & $\begin{array}{l}57.4 \\
60.0 \\
62.5 \\
50.0 \\
50.0\end{array}$ & $\begin{array}{l}75.2 \\
80.0 \\
80.0 \\
75.0 \\
64.3\end{array}$ & $\begin{array}{c}92.1 \\
100.0 \\
97.5 \\
37.5 \\
92.9\end{array}$ \\
\hline Overall & & 41.7 & 68.9 & 72.0 & 90.3 \\
\hline
\end{tabular}

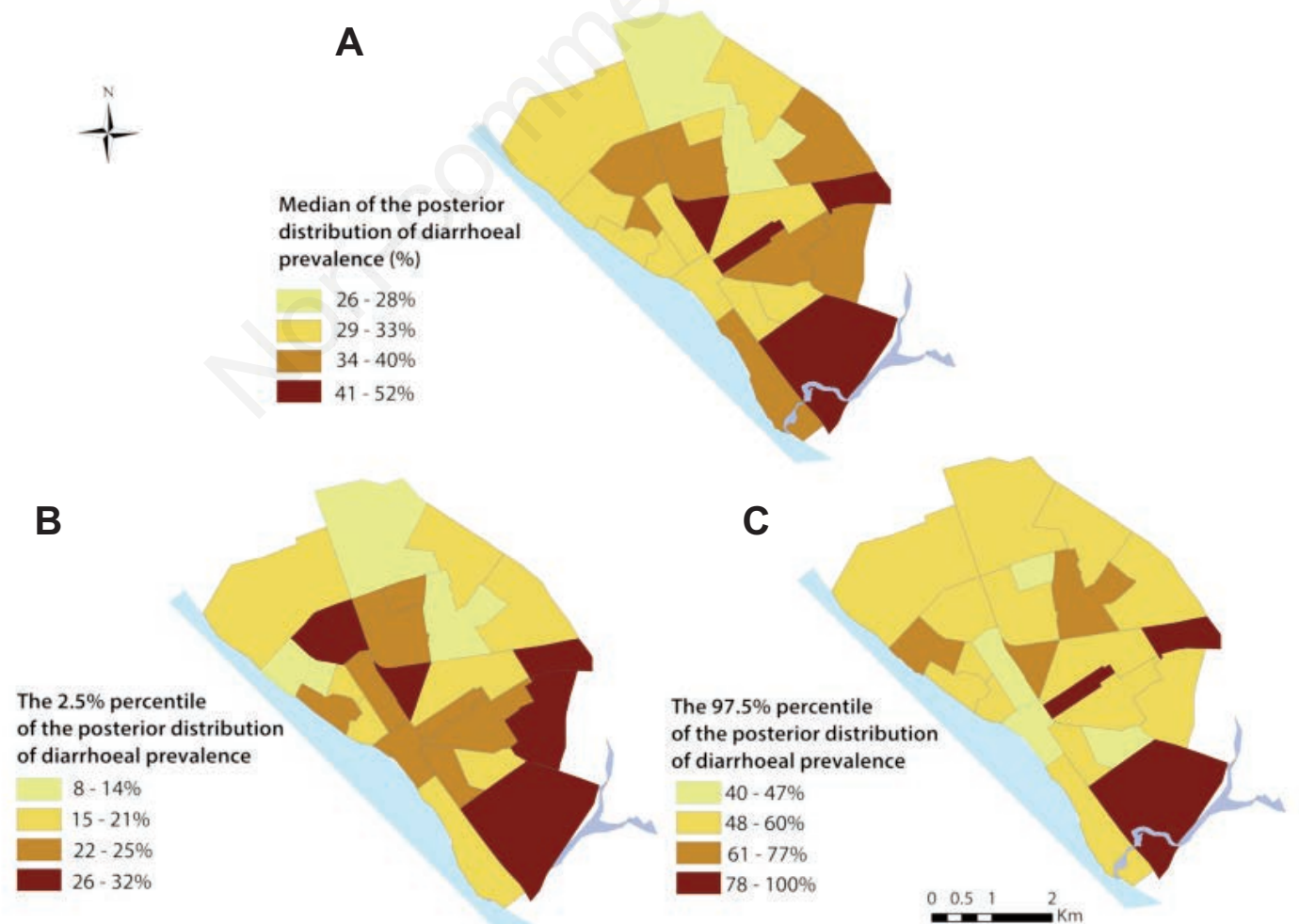

Figure 3. Spatial distribution of the smoothed diarrhoeal prevalence from the posterior median and the $2.5 \%$ and $97.5 \%$ percentiles. Median (A), 2.5\% percentile (B) and $97.5 \%$ percentiles (C) of the posterior distribution of diarrhoeal prevalence. Estimations are based on model 3. 
The findings from the Bayesian smoothed maps show substantial variation in the spatial distribution of diarrhoea with neighbourhoods of higher and lower than expected risk clustered. This might be explained by wider sociodemographic and economic inequalities between neighbourhoods (Osei and Stein, 2017b). Unsafe treated stored water and the high prevalence of diarrheal disease observed in the city of Mbour near the coastal area could be explained by the presence of many pathogens, which proliferate in brackish river and coastal water environments, along with the presence of higher levels of enteric microorganisms typically found in coastal areas. A similar pattern, with higher incidence of acute diarrhoea and cholera has been observed in India near the coastal areas (Alam et al., 2006; Kumar et al., 2016). Prior epidemiological studies have shown that people living in close proximity to coastal areas are at higher risk of contracting infectious diseases. This could be due to polluted coastal waters that may run inland during rainy season, leading to higher incidence of diarrhoea (Lipp et al., 2002; Rajendran et al., 2011; Jayakumar and Malarvannan, 2013).

Our study has several limitations that are offered for consideration. First, the study is based on morbidity data aggregated at a neighbourhood level. That is why we focus on modelling the spatially varying coefficient as realisations of a CAR process produced from the weighting of the burden in the neighbouring areas. Hence, it was not possible to account for local variations in neighbourhood covariate effects through spatially varying coefficients; and therefore we could not estimate neighbourhood-specific relative risk, which relies on point referenced data (Gelfand et al., 2003). Disease indices, such as the relative risk of common morbidity, are an important measure of estimate in disease mapping used for comparing neighbourhood health status for spatially explicit planning of health interventions (Osei and Stein, 2017b). Further studies using similarly rigorous statistical approaches as pursued here should map neighbourhood-specific relative risk estimates and determine the spatially varying association between the relative risk and potential risk factors.
Second, due to unavailability of temporal data, this study has also not considered a potential temporal variation of the prevalence of diarrhoea and possible temporal change in the sociodemographic, economic and climatic factors. The identification and understanding of clusters in space and time at neighbourhood level should be considered for future research.

Despite these limitations, the findings of the current study complement our prior research pertaining to the aetiology and local perceptions of diarrhoea in under 5-year-old children in Mbour (Thiam et al., 2019). The observed spatial pattern of diarrhoea provides valuable information for health programme managers to design and implement interventions. This spatially explicit information is not only important for the Ministry of Health, but also other ministries responsible for WASH aspects. Indeed, diarrhoea risk areas can, and should, be identified at neighbourhood level to tackle area-specific interventions, which might also be important for the control and prevention of suspected diarrhoea outbreaks.

\section{Conclusions}

Our findings revealed that childhood diarrhoea remains a major public health problem in the secondary city of Mbour, Senegal with considerable spatial heterogeneity from one neighbourhood to another. Our findings provide a deeper understanding of the geographical variation of neighbourhood health status and suggest that attention should be paid to children aged 2 years and above in the north and south peripheral neighbourhoods, such as Médine, Liberté, Grand Mbour, Zone Sonatel and Gouye Mouride, which are characterised by a lack of amenities such as availability of safe water source and improved sanitation. In such areas, priority attention would be important on WASH-related interventions in order to prevent and control diarrhoea in under 5-year-old children.

Table 4. Posterior median and 95\% Bayesian credible intervals of model 1 (model of diarrhoea risk based on climatic covariates), model 2 (model of diarrhoeal risk based on sociodemographic and economic covariates) and model 3 (model of diarrhoeal risk comprised climatic and sociodemographic and economic covariates).

\begin{tabular}{|c|c|c|c|}
\hline Model/variables & $\begin{array}{c}\text { Model } 1 \\
\text { RR (95\% BCI) }\end{array}$ & $\begin{array}{c}\text { Model } 2 \\
\operatorname{RR}(95 \% \text { BCI) }\end{array}$ & $\begin{array}{c}\text { Model } 3 \\
\text { RR (95\% BCI) }\end{array}$ \\
\hline \multicolumn{4}{|l|}{ Climatic variable } \\
\hline Altitude & $1.03(0.83-1.25)$ & & $0.79(0.63-0.96)^{*}$ \\
\hline Rainfall & $1.18(0.89-1.44)$ & & $1.40(1.10-1.74)^{*}$ \\
\hline $\mathrm{LST}_{d a y}$ & $1.07(0.89-1.16)$ & & $1.07(0.93-1.27)$ \\
\hline $\mathrm{LST}_{\text {night }}$ & $0.91(0.81-1.03)$ & & $0.82(0.69-1.03)$ \\
\hline \multicolumn{4}{|c|}{ Socioeconomic and environmental factors } \\
\hline udws & & $0.96(0.55-1.45)$ & $0.83(0.69-1.05)$ \\
\hline$u s d w$ & & $1.22(1.01-1.53)^{*}$ & $1.29(1.06-1.60)^{*}$ \\
\hline ulwd & & $0.89(0.78-1.05)$ & $0.96(0.86-1.12)$ \\
\hline uswd & & $1.03(0.78-1.30)$ & $1.06(0.78-1.43)$ \\
\hline Socioeconomic status & & $1.32(0.77-2.24)$ & $1.95(1.43-2.54) *$ \\
\hline
\end{tabular}

RR, relative risk; BCI, Bayesian credible interval; LST, land surface temperature; $u d w s$, unsafe drinking water source; $u s d w$, untreated stored drinking water; $u l w d$, unsafe liquid waste disposal; $u s w d$, unsafe solid waste disposal. *Statistically significant. 


\section{References}

Alam M, Hasan NA, Sadique A, Bhuiyan N, Ahmed KU, Nusrin S, Nair GB, Siddique AK, Sack RB, Sack DA, Huq A, Colwell RR, 2006. Seasonal cholera caused by Vibrio cholerae serogroups O1 and O139 in the coastal aquatic environment of Bangladesh. Appl Environ Microb 72:4096-104.

Bivand RS, Pebesma E, Gómez-Rubio V, 2013. Hello world: introducing spatial data. In: Bivand RS, Pebesma E, Gómez-Rubio V, eds. Applied spatial data analysis with R. Springer, New York, USA.

Black RE, Morris SS, Bryce J, 2003. Where and why are 10 million children dying every year? Lancet 361:2226-34.

Cressie N, 2015. Statistics for spatial data. John Wiley \& Sons, New York, USA.

Fischer Walker CL, Perin J, Aryee MJ, Boschi-Pinto C, Black RE, 2012. Diarrhea incidence in low- and middle-income countries in 1990 and 2010: a systematic review. BMC Public Health 12:220.

GBD 2015 DALYs and HALE Collaborators, 2016. Global, regional, and national disability-adjusted life-years (DALYs) for 315 diseases and injuries and healthy life expectancy (HALE), 1990-2015: a systematic analysis for the Global Burden of Disease Study 2015. Lancet 388:1603-58.

GBD 2016 Diarrhoeal Diseases Collaborators, 2018. Estimates of the global, regional, and national morbidity, mortality, and aetiologies of diarrhoea in 195 countries: a systematic analysis for the Global Burden of Disease Study 2016. Lancet Infect Dis 18:1211-28.

GBD Diarrhoeal Diseases Collaborators, 2017. Estimates of global, regional, and national morbidity, mortality, and aetiologies of diarrhoeal diseases: a systematic analysis for the Global Burden of Disease Study 2015. Lancet Infect Dis 17:909-48.

GBD 2017 Causes of Deaths Collaborators, 2018. Global, regional, and national age-sex-specific mortality for 282 causes of death in 195 countries and territories, 1980-2017: a systematic analysis for the Global Burden of Disease Study 2017. Lancet 392:1736-88.

Gelfand AE, Kim HJ, Sirmans C, Banerjee S, 2003. Spatial modeling with spatially varying coefficient processes. J Am Stat Assoc 98:387-96.

Institute for Health Metrics and Evaluation, 2016. The global burden of diseases, injuries, and risk factors study. Available from: http://healthdata.org/gbd Accessed: August 6, 2017.

Jayakumar K, Malarvannan S, 2013. Spatial mapping of cholera using GIS tools in Chennai, India. Arch Appl Sci Res 5:93-9.

Kumar VS, Devika S, George S, Jeyaseelan L, 2016. Spatial mapping of acute diarrheal disease using GIS and estimation of relative risk using empirical Bayes approach. Clin Epidemiol Global Health 5:87-96.

Lipp EK, Huq A, Colwell RR, 2002. Effects of global climate on infectious disease: the cholera model. Clin Microb Rev 15:757-70.

Osei FB, Stein A, 2017a. Diarrhea morbidities in small areas: accounting for non-stationarity in sociodemographic impacts using Bayesian spatially varying coefficient modelling. Sci Rep 7:9908.

Osei FB, Stein A, 2017b. Spatial variation and hot-spots of district level diarrhea incidences in Ghana: 2010-2014. BMC Public Health 17:617.

Prüss-Üstun A, Bartram J, Clasen T, Colford JM, JR, Cumming O, Curtis V, Bonjour S, Dangour AD, De France J, Fewtrell L, Freeman MC, Gordon B, Hunter PR, Johnston RB, Mathers C, Mäusezahl D, Medlicott K, Neira M, Stocks M, Wolf J, Cairncross S, 2014. Burden of disease from inadequate water, sanitation and hygiene in low- and middle-income settings: a retrospective analysis of data from 145 countries. Trop Med Int Health 19:894-905.

Rajendran K, Sumi A, Bhattachariya M, Manna B, Sur D, Kobayashi N, Ramamurthy T, 2011. Influence of relative humidity in Vibrio cholerae infection: a time series model. Indian J Med Res 133:138-45.

Sambe-Ba B, Espie E, Faye ME, Timbine LG, Sembene M, Gassama-Sow A, 2013. Community-acquired diarrhea among children and adults in urban settings in Senegal: clinical epidemiological and microbiological aspects. BMC Infect Dis 13:580.

Sire JM, Garin B, Chartier L, Fall NK, Tall A, Seck A, Weill FX, Breurec S, Vray M, 2013. Community-acquired infectious diarrhoea in children under 5 years of age in Dakar, Senegal. Paediatr Int Child Health 33:139-44.

Ssempiira J, Nambuusi B, Kissa J, Agaba B, Makumbi F, Kasasa S, Vounatsou P, 2017. Geostatistical modelling of malaria indicator survey data to assess the effects of interventions on the geographical distribution of malaria prevalence in children less than 5 years in Uganda. PLoS One 12:e0174948.

Thiam S, Diène AN, Fuhrimann S, Winkler MS, Sy I, Ndione JA, Schindler C, Vounatsou P, Utzinger J, Faye O, Cissé G, 2017a. Prevalence of diarrhoea and risk factors among children under five years old in Mbour, Senegal: a cross-sectional study. Infect Dis Poverty 6:109.

Thiam S, Fuhrimann S, Niang-Diène A, Sy I, Faye O, Utzinger J, Cissé G, 2017b. Urbanisation and its effect on risk factors associated with childhood diarrhoea in Mbour, Senegal: a visualisation. Geospat Health 12:632.

Thiam S, Sy I, Schindler C, Niang-Diène A, Faye O, Utzinger J, Cissé G, 2019. Knowledge and practices of mothers and caregivers on diarrhoeal management among under 5-year-old children in a medium-size town of Senegal. Acta Trop 194:155-64.

Walker CL, Rudan I, Liu L, Nair H, Theodoratou E, Bhutta ZA, O'Brien KL, Campbell H, Black RE, 2013. Global burden of childhood pneumonia and diarrhoea. Lancet 381:1405-16.

WHO, 2017. Diarrhoeal diseases: Fact sheet. World Health Organization, Geneva, Switzerland. Available from: http://www. who.int/mediacentre/factsheets/fs330/en/ Accessed: June 25, 2017. 